\title{
QUANTIFICAÇÃO DE GASES DE EFEITO ESTUFA EM ATERRO SANITÁRIO NO MUNICÍPIO DE CUIABÁ
}

\author{
QUANTIFICATION OF GREENHOUSE GASES IN LANDFILL IN CUIABÁ CITY
}

\author{
Ana Eveline Mendonça Mourato Limaa ${ }^{\text {, Osvaldo Borges Pinto Junior }}{ }^{\mathrm{a}}$ \\ aUniversidade de Cuiabá \\ anaevelinemourato@gmail.com, osvaldo.borges@gmail.com
}

Submissão: 08 de agosto de 2020 Aceitação: 19 de novembro de 2020

\section{Resumo}

A disposição do lixo no Brasil, onde o uso de "lixões" ainda é muito comum, é um dos graves problemas ambientais. A emissão de gases produzidos pela decomposição anaeróbia da matéria orgânica pode ser uma ameaça ao ambiente local causando danos à vegetação, gerando odores desagradáveis, oferecendo ainda riscos de explosão. Este estudo objetivou avaliar as emissões de dióxido de carbono $\left(\mathrm{CO}_{2}\right)$ e metano $\left(\mathrm{CH}_{4}\right)$ no Aterro Sanitário no Município de Cuiabá, utilizando câmaras estáticas. Avaliou três pontos de amostragem com diferentes tempos de deposição de resíduos. Os valores anuais de $\mathrm{CO}_{2}$ foram: no ponto $\mathrm{A}$ (deposição recente), $19,02 \mu \mathrm{g} \mathrm{CO}_{2} \mathrm{~m}^{-2} \mathrm{~h}^{-1}$; no ponto $\mathrm{B}$ (deposição intermediária), $4,36 \mu \mathrm{g} \mathrm{CO}_{2} \mathrm{~m}^{-2} \mathrm{~h}^{-1} \mathrm{e}$, no ponto $\mathrm{C}$ (deposição com maior tempo), 2,59 $\mu \mathrm{g} \mathrm{CO}_{2} \mathrm{~m}^{-2} \mathrm{~h}^{-1}$. A concentração de $\mathrm{CH}_{4}$ no ponto $\mathrm{A}$ foi $3,89 \mu \mathrm{g} \mathrm{CH}_{4} \mathrm{~m}^{-2} \mathrm{~h}^{-}$ 1 e 0,12 e $0,25 \mu \mathrm{g} \mathrm{CH}_{4} \mathrm{~m}^{-2} \mathrm{~h}^{-1}$ nos pontos $\mathrm{B}$ e $\mathrm{C}$, respectivamente. Os resultados de $\mathrm{CO}_{2}$ e $\mathrm{CH}_{4}$ tiveram comportamentos semelhantes, houve aumento das concentrações. O estudo concluiu que fluxo de gases é dependente do tempo de deposição de resíduos e que, de acordo com esse tempo de deposição, a compactação do solo atua como limitador na emissão de GEE.

Palavras-chave: Gerenciamento de resíduos sólidos; Mudanças climáticas; Fluxo de gases

\section{Abstract}

The disposal of garbage in Brazil is one of the serious environmental problems where the use of "dumps" is still very common. The emission of gases produced by the anaerobic decomposition of organic matter can be a threat to the local environment, causing damage to vegetation, generating unpleasant odors, also offering risks of explosion. This study objective evaluated emissions of Carbon Dioxide $\left(\mathrm{CO}_{2}\right)$ and Methane $\left(\mathrm{CH}_{4}\right)$ in landfill in the Cuiabá city, using static chambers. Three sampling points under different waste deposition times were evaluated. The annual flow of $\mathrm{CO}_{2}$ at point $A$ (recent deposition) was $19.02 \mu \mathrm{gO}_{2} \mathrm{~m}^{-2} \mathrm{~h}^{-1}$, at point $\mathrm{B}$ (intermediate), it was $4.36 \mu \mathrm{g} \mathrm{CO}_{2} \mathrm{~m}^{-2} \mathrm{~h}^{-1}$, and at point $\mathrm{C}$ (deposition with longer time) was $2.59 \mu \mathrm{g} \mathrm{CO}_{2} \mathrm{~m}^{-2} \mathrm{~h}^{\text {- }}$ 1. The concentration of $\mathrm{CH}_{4}$ at point $\mathrm{A}$ was $3.89 \mu \mathrm{g} \mathrm{CH}_{4} \mathrm{~m}^{-2} \mathrm{~h}$ and 0.12 and $0.25 \mu \mathrm{g} \mathrm{CH}_{4} \mathrm{~m}^{-2} \mathrm{~h}^{-1}$ respectively at points $\mathrm{B}$ and $\mathrm{C}$. The results of $\mathrm{CO}_{2}$ and $\mathrm{CH}_{4}$, have similar behavior, occurring increase of concentrations. It concluded that gas flow is dependent on the time of waste deposition, and according to the time of deposition, the soil compaction performances as a limiting factor in GHG emissions.

Keywords: Solid-waste management; Change Climatic; Gas flow

\section{INTRODUÇÃO}

Os gases produzidos pelo lixo nos municípios brasileiros contribuem significativamente para 0 efeito estufa. Devido à composição aeróbia e anaeróbica desse lixo há emissão de gases de efeito estufa. A emissão de gases tende a agravarse com 0 aumento do consumo de bens descartáveis, que passam cada vez mais a compor os grandes volumes de lixo gerados pela população. A maioria das emissões provém dos aterros sanitários e lixões, que continuam sendo o 
principal destino para descarte de resíduos (LOU; NAIR, 2009).

No ano de 1991, o Painel Intergovernamental sobre Mudanças Climáticas (IPCC) das Nações Unidas publicou seu primeiro relatório analisando o aumento da temperatura terrestre em decorrência da intensificação do efeito estufa. Esse relatório passou a ser considerado a principal referência sobre o assunto mudança climática global. Na referida publicação, intitulada Climate Change - The IPCC Scientific Assessment, pela primeira vez o IPCC defendeu a tese de que a concentração de gases de efeito estufa estava aumentando na atmosfera global em consequência das emissões antrópicas. O termo "efeito estufa" se aplica ao aumento significativo da temperatura média global do ar em vários graus como resultado da elevação da concentração de gás carbônico e de outros gases na atmosfera (BAIRD, 2002).

Os três principais gases do efeito estufa são - dióxido de carbono $\left(\mathrm{CO}_{2}\right)$, que é o mais abundante dos GEE (Gases de Efeito Estufa), sendo produzido principalmente pela queima de combustíveis fósseis e remoção de florestas, contribuindo com $56 \%$ para o valor total das emissões; o metano $\left(\mathrm{CH}_{4}\right)$, que é subproduto da digestão anaeróbia da matéria orgânica e é gerado em aterros sanitários, aterros controlados, lixões, na criação de gado e no cultivo de arroz, contribuindo com $16 \%$ para o valor total das emissões; e o óxido nitroso $\left(\mathrm{N}_{2} \mathrm{O}\right)$, que é resultante da queima de combustíveis fósseis e de alguns processos industriais, contribuindo com $5 \%$ para o valor total das emissões (INTERGOVERNMENTAL PANEL ON CLIMATE CHANGE, 2007).

Os gases provenientes dos locais de disposição de resíduos orgânicos nos aterros sanitários são resultados de diversos fatores e processos: físicos, químicos e biológicos. Em sua maior parte, esses gases são: o metano (entre 50 e $60 \%$ ) e o dióxido de carbono (40 e $50 \%$ ) (SCHEUTZ; KJELDSEN, 2019).

Os resíduos sólidos urbanos apresentam diversas características, principalmente pela heterogeneidade dos fatores que contribuem para a sua geração: o número de habitantes do local, as condições climáticas, os hábitos e costumes da população, o nível cultural dos habitantes, as variações sazonais, o poder aquisitivo da população, a taxa de eficiência do projeto ou do programa de coleta, o tipo de equipamento de coleta e as leis e regulamentações específicas (BUENROSTRO; BOCCO, 2003; OJEDABENITEZ; VEGA; RAMIREZ-BARRETO, 2003; METIN; ERZTURK; NEYIM, 2003). Influenciam nas características dos resíduos sólidos urbanos variáveis como: condições ambientais e políticas, número de habitantes, nível educacional, poder aquisitivo, hábitos e costumes da população (RAMOS et al., 2018).

No Brasil, uma parte do resíduo sólido urbano destina-se aos aterros sanitários, que são caracterizados como uma forma de disposição dos resíduos sólidos no solo, a qual, fundamentada em critérios de engenharia e procedimentos operacionais, permite o confinamento seguro. Isso garante o controle da poluição ambiental e minimiza os impactos ambientais (BOSCOV, 2008). O aterro sanitário é uma das formas de tecnologias mais utilizadas para descarte dos resíduos sólidos urbanos, pois a sua instalação e manutenção são relativamente simples, além de terem menos custos operacionais (COLVERO; GOMES; PFEIFFER, 2015).

O aterro do Município de Cuiabá, no ano de 2015, recebeu 332.089,07 toneladas de resíduos sólidos urbanos e, em 2016, 231.163,70 toneladas de resíduos sólidos urbanos, cerca de 781 toneladas por dia, de acordo com o Sistema Nacional de Informações sobre Saneamento (MINISTÉRIO DAS CIDADES, 2015, 2016). Esse aterro foi classificado como controlado, porém não apresenta medidas de controle e gerenciamento dos gases resultante das emissões.

Os estudos que visam contribuir com dados sobre os gases lançados na atmosfera são essenciais para validar as tecnologias para mitigar essas emissões (NOGUEIRA et al., 2015). Quantificar e avaliar a dinâmica dessas emissões tornará possível a organização de projetos futuros para seu controle e posterior gerenciamento como, por exemplo: a utilização do gás emitido para aquecimento direto, para geração de energia elétrica em células de combustíveis e para recuperação calorífica através de queimadores no aterro. Com essas alternativas, consegue-se diminuir as emissões e ter a oportunidade de vender créditos de carbono, atingindo assim metas como as estabelecidas no Protocolo de Quioto.

Esta pesquisa teve por objetivo quantificar as emissões de dióxido de carbono $\left(\mathrm{CO}_{2}\right)$ e metano $\left(\mathrm{CH}_{4}\right)$ em diferentes tempos de deposição no aterro sanitário no Município de Cuiabá. 


\section{METODOLOGIA}

\section{Localização e características gerais}

O estudo foi realizado no Aterro Municipal da Cidade de Cuiabá, localizado na parte nordeste da área urbana da cidade, na Estrada Balneário Letícia, entre os Bairros do Centro Político Administrativo (CPA) e a Rodovia Emanuel Pinheiro, com latitude $15^{\circ} 35^{\prime} 12^{\prime \prime} S$ e longitude $56^{\circ} 04^{\prime} 16^{\prime \prime}$ W e com uma área de 15,16 ha. Situado na região da Baixada Cuiabana, esse aterro possui uma camada de solo, em geral, pouco espessa, e logo se encontram rochas do Grupo Cuiabá. $\mathrm{Na}$ área do estudo, particularmente, encontra-se a Formação Miguel Sutil, composta por filitos e metarenitos, com fraturas preenchidas por quartzo (MIGLIORINI, 1999). O clima da região, segundo a classificação de Köppen, é do tipo tropical de savana - Aw, quente e úmido, com uma estação seca mais prolongada e uma estação úmida de quatro meses, entre dezembro e março, com temperatura média de $25^{\circ} \mathrm{C}$ e precipitação média anual de $1500 \mathrm{~mm}$.

\section{Delineamento experimental e pontos de coleta}

$O$ delineamento experimental foi realizado aleatoriamente, os pontos foram escolhidos considerando-se as áreas com diferentes períodos de deposição no aterro sanitário (Figura 1). Os resíduos são depositados diariamente. $O$ ponto $A$, localizado na parte mais baixa do aterro, é a parte mais recente em termos de deposição de resíduos (2 meses). O ponto B foi selecionado por ser uma área intermediária de deposição, tendo aproximadamente dez anos. Neste ponto, há deposição dos resíduos que antes se encontravam na parte externa do aterro, ou seja, que são provenientes de realocação. Porém, esta é uma área que se encontra quase que em sua totalidade já paralisada. $O$ ponto $C$ foi selecionado na parte mais antiga do aterro, com aproximadamente 18 anos, ocupando a área mais alta, onde não ocorre mais deposição de resíduos, havendo somente compactação ou, ocasionalmente, cobertura do que já está depositado no local.

\section{Descrição dos equipamentos}

Foi utilizado um termômetro digital, modelo Fluke II para medidas de temperatura do solo $\left({ }^{\circ} \mathrm{C}\right)$, composto por uma sonda de $11 \mathrm{~cm}$ e com precisão de $\pm 0,1^{\circ} \mathrm{C}$; um termo higrômetro digital int./ext., Incoterm, modelo 7666.02.0.00, com precisão de \pm $0,1^{\circ} \mathrm{C}$, para medir a temperatura interna da câmara de amostragem e a umidade relativa do ar (\%).

\section{Figura 1 - Localização do Aterro Municipal de Cuiabá}

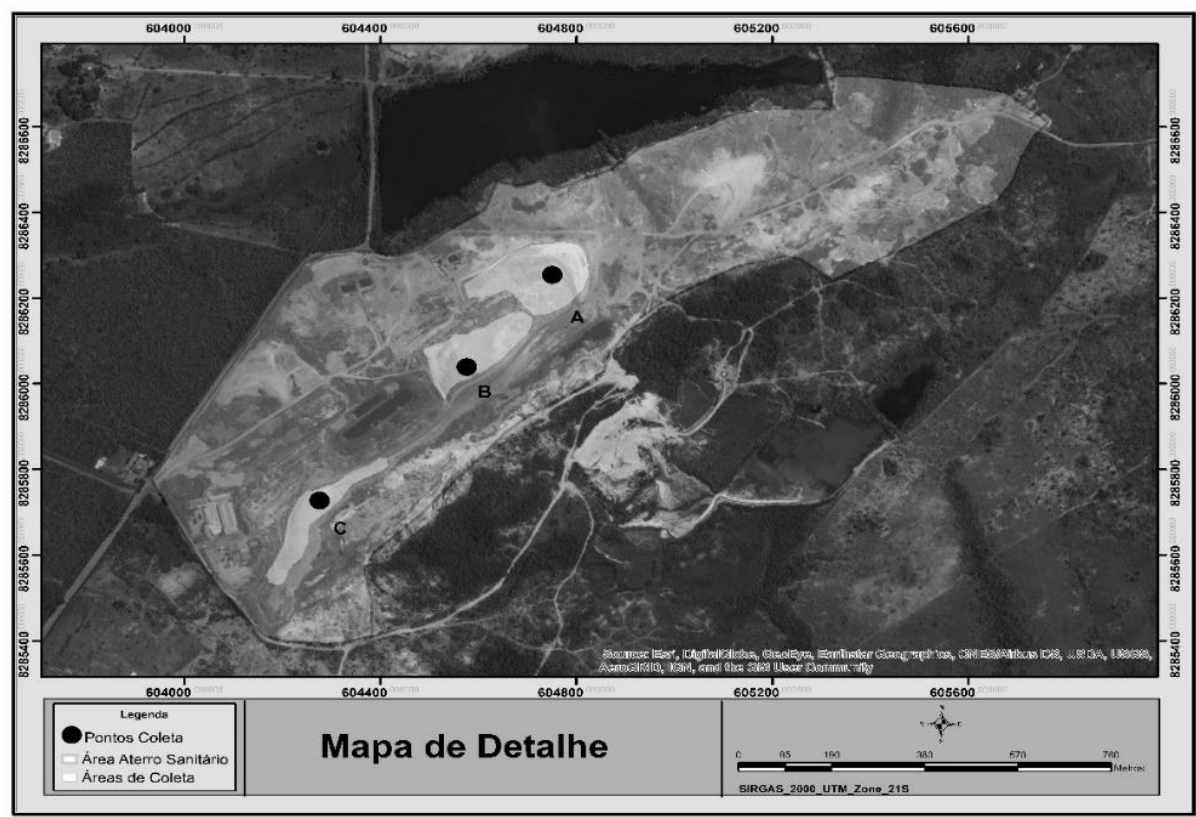

A - Recente; B - Intermediária; C - Antiga

Fonte: Autor. 


\section{Medidas de $\mathrm{CO}_{2}$ e $\mathrm{CH}_{4}$ por cromatografia gasosa}

A coleta das amostras de gases foi realizada através do método de câmaras estáticas (MOSIER; DELGADO; KELLER, 1998). A câmara é fechada, restringindo a passagem do gás liberado através do solo para atmosfera, de modo que, havendo mudanças na composição das amostras, elas possam ser medidas. Sobre a camada de deposição, foram fixadas bases metálicas no solo a uma profundidade de $5 \mathrm{~cm}$, acopladas a bacias de plástico de $\approx 2$ litros $e$ revestidas por mantas térmicas (Figura 2). $\mathrm{Na}$ parte superior das câmaras, foram instaladas válvulas pelas quais eram retiradas amostras dos gases.

Figura 2 - Bacia de plástico coberta com manta térmica (A); Bacia de plástico coberta com manta térmica, com termo higrômetro para leitura da temperatura e umidade da câmara (B); Representação da dimensão das bacias de plástico (C).
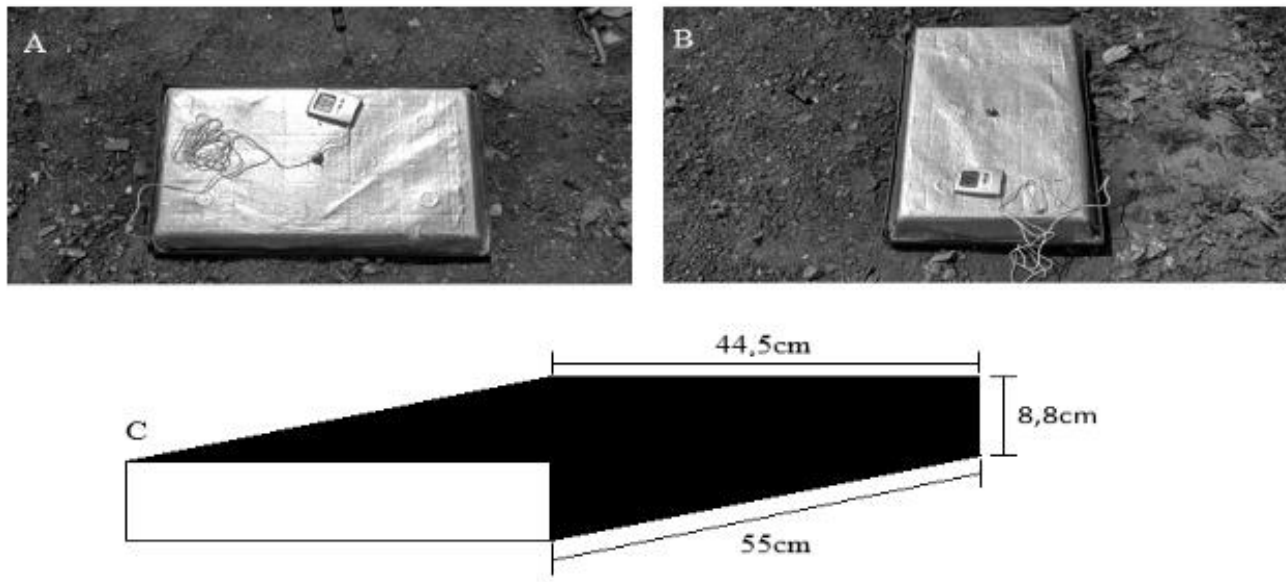

Foram realizadas campanhas com frequência bimestral, de agosto de 2015 a julho de 2016. As coletas ocorreram no período da manhã, em triplicada, entre 9:00 h e 11:00 h, horário representativo dos fluxos médios diários de gases de efeito estufa.

A coleta de amostras dos gases foi realizada utilizando-se uma seringa de plástico de $20 \mathrm{~mL}$ com agulha hipodérmica, em quatro tempos após a inserção da câmara sobre o solo. A primeira medida foi realizada no primeiro minuto após a colocação da placa e, depois, em intervalos de 20 minutos até decorrer o tempo de 60 minutos. As seringas utilizadas tinham acopladas válvulas plásticas de três vias, que asseguravam a sua vedação entre os intervalos de coleta das amostras.

Concomitantemente foram coletadas temperaturas do ar e do solo. As amostras foram armazenadas em ampolas gasométricas de vidro com capacidade de $40 \mathrm{~mL}$, metodologia adaptada de Rodrigues e Mello (2012).
Após a coleta, as amostras foram enviadas para o Laboratório de Cromatografia da Embrapa Agrossilvipastoril, em Sinop-MT. As análises foram feitas por cromatografia gasosa utilizando-se colunas empacotadas em temperatura de $70^{\circ} \mathrm{C}$. Foi usado um cromatógrafo modelo GC 2014 Greenhouse (Shimadzu, Japão) equipado com um detector de captura de elétrons (ECD) para análise.

A variação da concentração de $\mathrm{CO}_{2}, \mathrm{CH}_{4} \mathrm{e}$ $\mathrm{N}_{2} \mathrm{O}$ nas câmaras foi calculada através do coeficiente angular da equação da reta ajustada (BENDER et al., 2020), utilizando-se a seguinte equação:

$$
\text { Emissão de GEE }=\frac{\Delta \mathrm{GEE}}{\Delta \mathrm{t}} \cdot \frac{\mathrm{V}}{\mathrm{A}} \cdot \mathrm{m} \cdot \frac{\mathrm{P}}{\mathrm{T} \cdot \mathrm{R}}
$$

Em que:

$\triangle G E E / \Delta t$ é a variação da concentração de cada GEE no interior da câmara no tempo da coleta ( $\mu$ $\left.\mathrm{mol}^{-1} \mathrm{~h}^{-1}\right)$, 
V é o volume da câmara $\left(\mathrm{m}^{3}\right)$,

A é a área da câmara $\left(\mathrm{m}^{2}\right)$,

$\mathrm{m}$ é o peso molecular do composto $\left(\mathrm{g} \mathrm{mol}^{-1}\right)$,

$P$ é a pressão atmosférica (atm),

T é a temperatura do solo em Kelvin $(\mathrm{K}) \mathrm{e}$

$R$ é a constante dos gases ideais $\left(0,082\right.$ atm $\mathrm{L} \mathrm{mol}^{-}$ $\left.{ }^{1} \mathrm{~K}^{-1}\right)$.

As concentrações de $\mathrm{CH}_{4}, \mathrm{CO}_{2}$ e $\mathrm{N}_{2} \mathrm{O}$ foram expressas em $\mu \mathrm{g} \mathrm{m}^{-2} \mathrm{~h}^{-1}$.

\section{RESULTADOS}

\section{Fluxo de $\mathrm{CO}_{2}$}

O fluxo de $\mathrm{CO}_{2}$ apresentou as maiores concentrações médias no mês de outubro/15 e em março/16. Observou-se que o ponto A (deposição recente) foi $O$ que apresentou as maiores amplitudes $\left(4,51 \mu \mathrm{g} \mathrm{CO}_{2} \mathrm{~m}^{-2} \mathrm{~h}^{-1}\right.$ e $11,1 \mu \mathrm{g} \mathrm{CO} \mathrm{Cm}^{-2}$ $\left.\mathrm{h}^{-1}\right)$. A média anual do fluxo de $\mathrm{CO}_{2}$ no ponto $A$ foi $3,17 \mu \mathrm{g} \quad \mathrm{CO}_{2} \mathrm{~m}^{-2} \mathrm{~h}^{-1}$. A deposição recente $\mathrm{e}$ recorrente no ponto $A$ favorece a hidrólise e a decomposição aeróbia e a consequente formação de $\mathrm{CO}_{2}$.

A média do fluxo de $\mathrm{CO}_{2}$ no ponto $\mathrm{B}$ foi 0,72 $\mu \mathrm{g} \mathrm{CO}_{2} \mathrm{~m}^{-2} \mathrm{~h}^{-1}$; e a média do ponto $\mathrm{C}$ foi $0,43 \mu \mathrm{g}$ $\mathrm{CO}_{2} \mathrm{~m}^{-2} \mathrm{~h}^{-1}$ (Figura 3). A concentração de $\mathrm{CO}_{2}$ apresentou-se de forma crescente nas primeiras coletas, sendo que a máxima ocorreu na quarta coleta; após esse pico houve um decréscimo constante das concentrações até o final do período de estudo.

Figura 3 - Fluxo de $\mathrm{CO}_{2}$ no aterro, nos períodos de coleta.

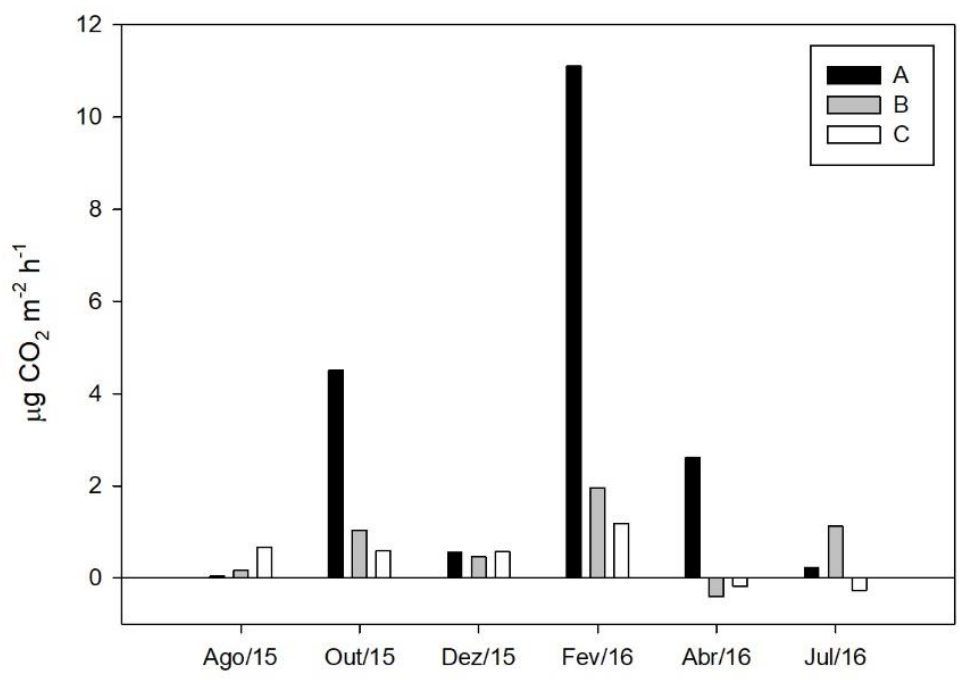

(Tempos de deposição: A - Recente; B - Intermediária; C - Antiga)

$\mathrm{Na}$ figura 4, são mostradas as áreas de monitoramento do fluxo de $\mathrm{CO}_{2}$. Observa-se que o ponto $\mathrm{A}$ foi a área onde foram obtidas as maiores médias e amplitudes, seguidas das do ponto $B$ e C. Esses valores corroboram a hipótese do resultado que seria esperado, pois o tempo de deposição distinto entre as áreas é o fator mais significativo de controle do fluxo de $\mathrm{CO}_{2}$. Segundo Ignatius (2005), em corpos de prova submetidos à saturação de água a partir de $80 \%$, há uma continuidade de água e o fluxo advectivo do ar no solo se anula, dificultando também o difusivo, pois este deve ser dissolvido no líquido.

\section{Fluxo de $\mathrm{CH}_{4}$}

A maior amplitude dos fluxos de $\mathrm{CH}_{4}$ durante as coletas foi observado no ponto $A$, que, como justificado anteriormente, é o ponto onde o aterro é mais recente e por isso existe maior revolvimento e deposição de material, o que favorece a amplitude dos fluxos desse gás. Durante o período do estudo, a média do fluxo de $\mathrm{CH}_{4}$ no ponto A foi $3,89 \mu \mathrm{g} \mathrm{CH}_{4} \mathrm{~m}^{-2} \mathrm{~h}^{-1}$; no ponto $\mathrm{B}$, foi $0,12 \mu \mathrm{g} \mathrm{CH}_{4} \mathrm{~m}^{-2} \mathrm{~h}^{-1}$; e no ponto $\mathrm{C}$, foi $0,25 \mu \mathrm{g}$ $\mathrm{CH}_{4} \mathrm{~m}^{-2} \mathrm{~h}^{-1}$ (Figura 5).

Assim como a concentração de $\mathrm{CO}_{2}, \circ \mathrm{CH}_{4}$ apresenta-se de forma crescente nas primeiras coletas, a concentração máxima durante o período 
de monitoramento foi observada na quarta coleta, havendo um decréscimo constante das concentrações até o final do estudo (Figura 6).

A amplitude observada no ponto A (Figura

6) deve-se ao tempo de deposição. Portando, devido ao tempo deposição (mais recente quando comparado aos demais), tem-se a formação de gases em maior concentração, justificando os valores superiores de $\mathrm{CH}_{4}$ encontrados.

\section{Figura 4 - Fluxos de $\mathrm{CO}_{2}$ observados nas áreas de deposição.}

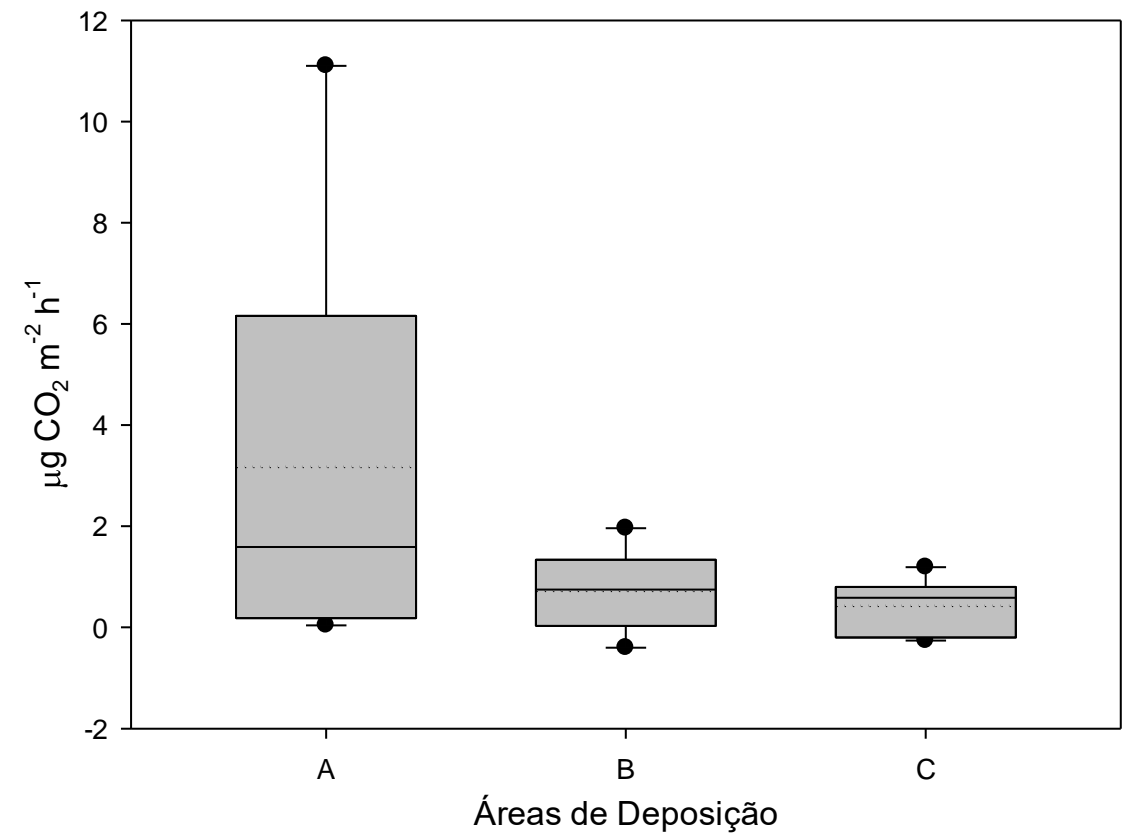

(Tempos de deposição: A - Recente; B - Intermediária; C - Antiga)

(Linha tracejada representa a mediana dos dados)

\section{Figura 5 - Fluxo de $\mathrm{CH}_{4}$ no aterro nos períodos de coleta}

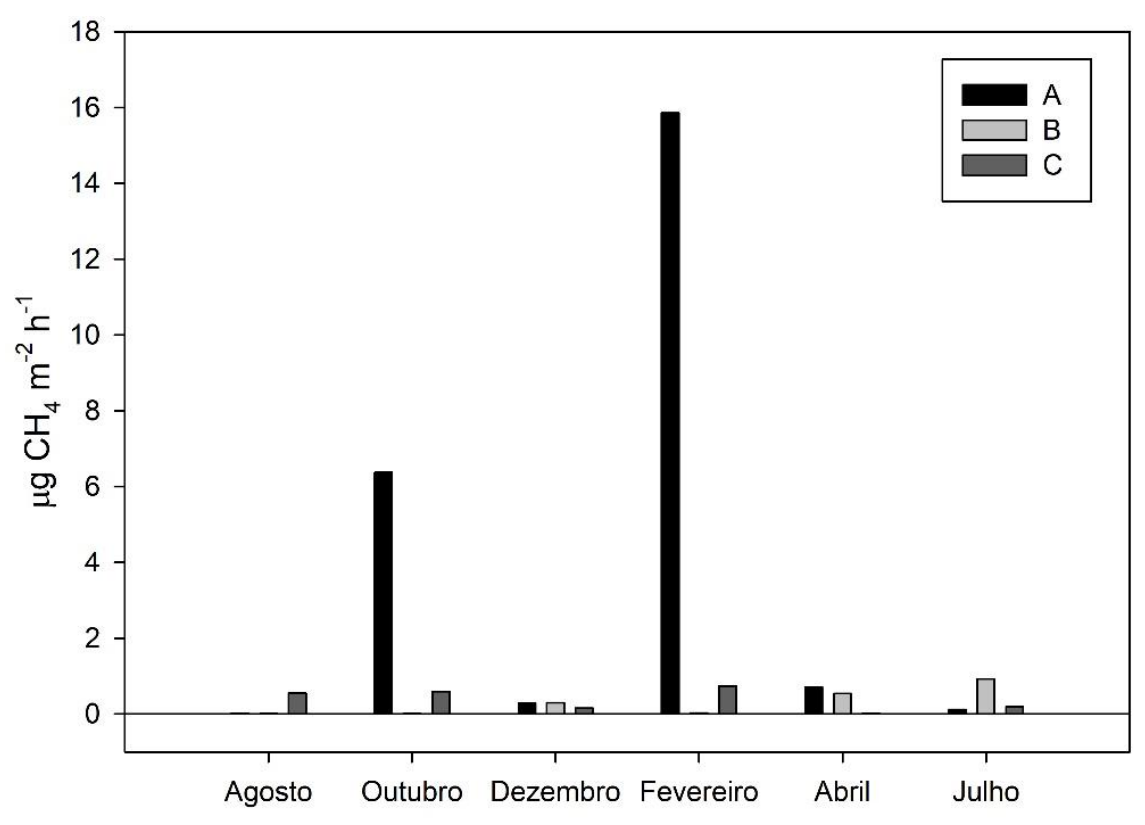

(Tempos de deposição: A - Recente; B - Intermediária; C - Antiga) 


\section{Figura 6 - Fluxos de $\mathrm{CH}_{4}$ observados nas áreas de deposição}

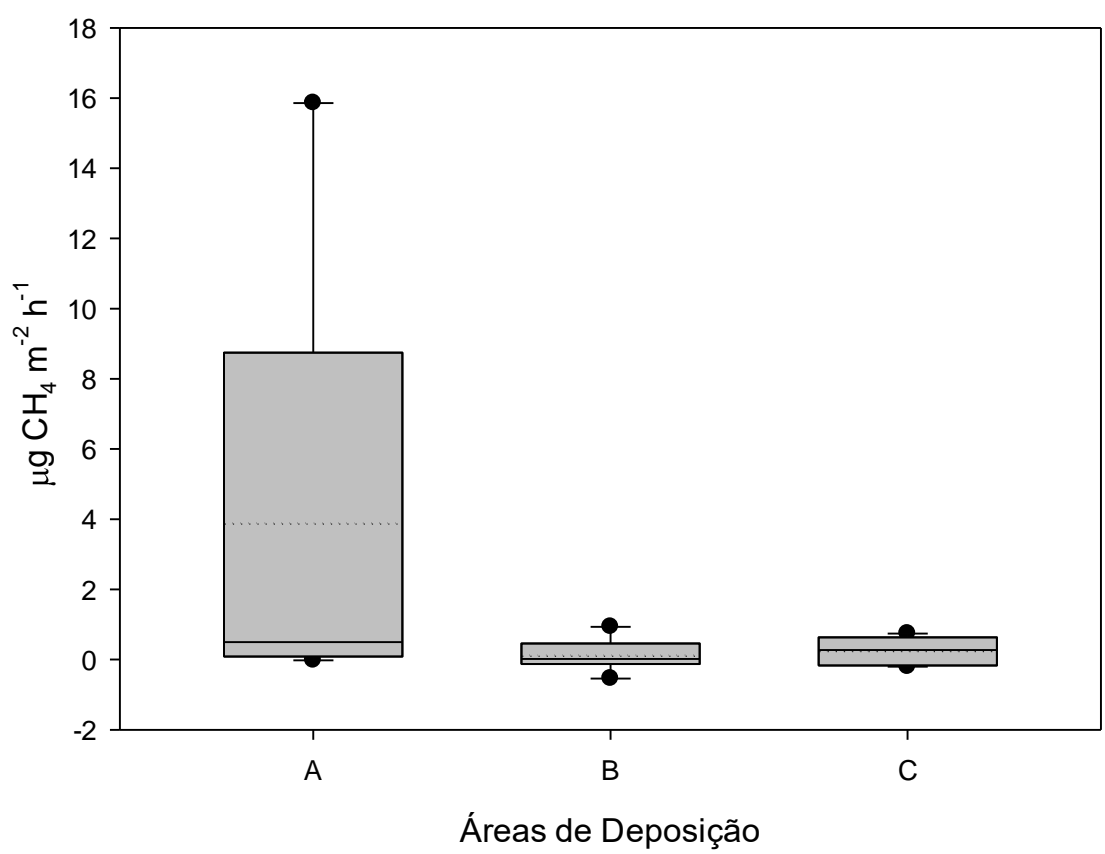

(Tempos de deposição: A - Recente; B - Intermediária; C - Antiga) (Linha tracejada representa a mediana dos dados)

Qian, Koerner e Gray (2003) descreveram que a geração do metano é dependente de duas variáveis temporais: lag time (período da disposição dos resíduos até o início da geração de metano) e conversion time (período da disposição do resíduo até o fim da geração do metano). Observa-se que o ponto A tem um lag time mais curto, o que torna a difusão do gás mais rápida se comparada aos demais pontos. A compactação do solo nos pontos $\mathrm{B}$ e $\mathrm{C}$ dificulta o transporte do gás para atmosfera.

De uma forma geral, os resultados de $\mathrm{CO}_{2} \mathrm{e}$ $\mathrm{CH}_{4}$ foram semelhantes, ocorrendo aumento das suas concentrações. A ampla faixa de variação do fluxo de $\mathrm{CH}_{4}$ e $\mathrm{CO}_{2}$ indica que existem vários fatores que interferem na emissão dos gases, desde a qualidade e espessura da camada de cobertura do solo até as características geotécnicas deste (tipo, grau de compactação e de saturação), pressões de gases no contato soloresíduo, idade dos resíduos, condições climáticas da região, entre outros (MARIANO; JUCÁ, 2011). $O$ decréscimo no valor do fluxo de metano e dióxido de carbono pode ocorrer devido à ocupação pela água de chuva dos poros presentes na camada de cobertura do solo, fenômeno observado em experimentos florestais e em medidas de concentração de gases.

Os resultados encontrados nesta pesquisa mostram que houve emissão de $0,0001 \mathrm{~g} \mathrm{CH}_{4} \mathrm{~m}^{2}$ dia a $0,0004 \mathrm{gCH}_{4} \mathrm{~m}^{2}$ dia, valores próximos aos encontrados por Akerman e colaboradores (2007), quais sejam, entre $0,0004 \mathrm{~g}$ a $0,43 \mathrm{~m}^{2}$ dia. Os autores também utilizaram uma câmara estática e trabalharam com resíduos com idade 0 a 24 anos, o que condiz com o aterro de Cuiabá, que tem 20 anos de deposição de resíduos.

\section{CONCLUSÃO}

Os mecanismos de transporte dos gases variam conforme as condições operacionais do aterro e do solo onde os resíduos são aplicados, sendo possível considerar que os processos de advecção se sobrepõem aos de difusão. No aterro estudado, o fluxo é baixo, pelo menos na sua superfície, indicando que a compactação do solo, em conjunto com as suas próprias características naquela área, atua como um limitador na emissão de gases para atmosfera. A dinâmica de gases de efeito estufa é muito inconstante, por essa razão recomenda-se que estudos para 0 seu monitoramento devam ser realizados pelo menos a cada 5 anos, especialmente em áreas de 
vulnerabilidade ambiental, como é o caso dos aterros sanitários.

\section{Agradecimentos}

Os autores do trabalho agradecem a Universidade de Cuiabá (UNIC), a Fundação Nacional de Desenvolvimento do Ensino Superior Particular (Funadesp), pelo apoio financeiro concedido e a Embrapa Agrossilvipastoril por contribuir na realização das análises laboratoriais desta pesquisa.

\section{REFERÊNCIAS}

AKERMAN, A. et al. Methane emissions measurements on different landfills. In: INTERNATIONAL WASTE MANAGEMENT AND LANDFILL SYMPOSIUM, 11. 2007, Sardinia, Italy. Anais [...] Cagliari, Italy: Environmental Sanitary Engineering Centre (CISA), 2007. p.10.

BAIRD, C. Química Ambiental. Trad. Maria A. L. Recio e Liz C. M. Carrera. 2. ed. Porto Alegre: Bookman, 2002.

BENDER, D. D. B. B. et al. Emissão de Óxido Nitroso e Metano em Solo de Campo Natural Convertido em Silvicultura. In: SALÃO INTERNACIONAL DE ENSINO, PESQUISA E EXTENSÃO SÃO PAULO, 8., 2016, Uruguaiana. Anais [...] Curitiba: Unipampa, 2020. (v.8, n. 4). Disponível em:

$<$ https://periodicos.unipampa.edu.br/index.php/SIEPE/a rticle/view/85321 >. Acesso em: 03 dez. 2018.

BOSCOV, M. E. G. Geotecnia ambiental. São Paulo: Oficina de Textos, 2008.

BUENROSTRO, O.; BOCCO, G. Solid waste management in municipalities in Mexico: goals and perspectives. Resources, conservation and recycling, v. 39, n. 3, p. 251-263, 2003.

COLVERO, D. A.; GOMES, A. P. D; PFEIFFER, S. C. Análise dos custos das rotas tecnológicas dos resíduos sólidos urbanos de Cidade Ocidental, Goiás. Revista Sodebras, v. 10, n. 117, p. 196-204, 2015.

IGNATIUS, S. G. Fluxo advectivo de gás em solos determinação dos parâmetros em laboratório. In: CONGRESSO BRASILEIRO DE MECÂNICA DOS SOLOS E ENGENHARIA GEOTÉCNICA, 13., 2005, Curitiba. Anais [...] Curitiba-PR: Associação Brasileira de Mecânica dos Solos e Engenharia Geotécnica, 2005.

INTERGOVERNMENTAL PANEL on CLIMATE
CHANGE. Climate change: 2007.The IPCC response strategies. Synthesis Report, 2007. Disponível em: <https://www.ipcc.ch/report/ar4/syr/> Acesso em: 07 dez. 2018.

LOU, X. F.; NAIR, J. The impact of landfilling and composting on greenhouse gas emissions - A review. Bioresource Technology, 100, p. 3792-3798, 2009.

MARIANO, M. O. H.; JUCÁ, J. F. T. Eficiência das camadas de cobertura na retenção de emissões de biogás em aterros sanitários: estudo de caso em Olinda, PE, Brasil. Revista Águas \& Resíduos, v. 15, p. 26-31, 2011.

METIN, E., ERZTURK, A., NEYIM, C. Solid waste management practices and review of recovery and recycling operations in Turkey. Waste Management, v. 23(5), p. 425-432, 2003.

MIGLIORINI, R.B. Hidrogeologia em meio urbano: região de Cuiabá e Várzea Grande - MT. 1999. 146 f. Tese (Doutorado em Geologia) - Instituto de geociências, Universidade de São Paulo (USP), São Paulo, 1999.

MOSIER, A. R.; DELGADO, J. A.; KELLER, M. Methane and nitrous oxide fluxes in an acid Oxisol in western Puerto Rico: effects of tillage, liming and fertilization. Soil Biology and Biochemistry, v. 30, n. 14, p. 2087-2098, 1998

NOGUEIRA, A. K. D. S. et al. Emissões de óxido nitroso e metano do solo em áreas de recuperação de pastagens na Amazônia Matogrossense. Química Nova, v. 38, n. 7, p. 937-943, 2015.

OJEDA-BENITEZ, S.; VEGA, C. A.; RAMIREZBARRETO, M. E. Characterization and quantification of household solid wastes in a Mexican city. Resources, Conservation and Recycling, v. 39, n. 3, p. 211-222, 2003.

QIAN, X.; KOERNER, R. M.; GRAY, D. H. Translational failure analysis of landfills. Journal of Geotechnical and Geoenvironmental Engineering, v. 129, n. 6, p. 506-519, 2003.

RAMOS, P. V. T. A. et. al. A gestão ambiental: melhoria do processo produtivo no tratamento de resíduos sólidos urbanos com recuperação energética. Brazilian Journal of Development, Curitiba, v. 4, n. 5, p. 2081 2096, 2018.

RODRIGUES, R. D. A. R.; MELLO, W. Z. D. Fluxos de óxido nitroso em solos com cobertura de floresta ombrófila densa montana na Serra dos Órgãos, Rio de Janeiro. Química Nova, v. 35, n. 8, p. 1549-1553, 2012. 
SCHEUTZ, C.; KJELDSEN, P. Guidelines for landfill gas emission monitoring using the tracer gas dispersion method. Waste Management, v. 85, p. 351-360, 2019.

MINISTÉRIO DAS CIDADES. Secretaria Nacional de Saneamento Ambiental. Sistema Nacional de Informações sobre Saneamento. Diagnóstico do
Manejo de Resíduos Sólidos Urbanos. Brasília, DF, 2015.

MINISTÉRIO DAS CIDADES. Secretaria Nacional de Saneamento Ambiental. Sistema Nacional de Informações sobre Saneamento. Diagnóstico do Manejo de Resíduos Sólidos Urbanos. Brasília, DF, 2016. 